\title{
Weak Measurable Optimal Controls for the Problems of Bolza
}

\author{
Gerardo Sánchez Licea
}

check for

updates

Citation: Sánchez Licea, G. Weak Measurable Optimal Controls for the Problems of Bolza. Mathematics 2021, 9, 191. https://doi.org/10.3390/ math9020191

Received: 21 December 2020 Accepted: 13 January 2021 Published: 19 January 2021

Publisher's Note: MDPI stays neutral with regard to jurisdictional clai$\mathrm{ms}$ in published maps and institutional affiliations.

Copyright: () 2021 by the author. Licensee MDPI, Basel, Switzerland. This article is an open access article distributed under the terms and conditions of the Creative Commons Attribution (CC BY) license (https:// creativecommons.org/licenses/by/ $4.0 /)$.
Departamento de Matemáticas, Facultad de Ciencias, Universidad Nacional Autónoma de México, Ciudad de México 04510, Mexico; gesl@ciencias.unam.mx

\begin{abstract}
Two sufficiency theorems for parametric and a nonparametric problems of Bolza in optimal control are derived. The dynamics of the problems are nonlinear, the initial and final states are free, and the main results can be applied when nonlinear mixed time-state-control inequality and equality constraints are presented. The deviation between admissible costs and optimal costs around the optimal control is estimated by functionals playing the role of the square of some norms.
\end{abstract}

Keywords: calculus of variations; optimal control; mixed restraints; inequality and equality restrictions; free initial and final states; sufficient conditions; weak minima; measurable optimal controls

MSC: 49K15

\section{Introduction}

In this paper, we derive two new sufficiency theorems in optimal control problems as the parametric and nonparametric problems of Bolza with nonlinear dynamics, free initial and final states, and inequality and equality mixed time-state-control constraints. The fundamental components of the sufficiency theorems of this article are a similar version of the Pontryagin maximum principle, a hypothesis usually called the transversality condition, a crucial second order inequality arising from the original algorithm employed to prove one of the sufficiency theorems, a related hypothesis of the Legendre-Clebsh necessary condition, the positivity of a quadratic function on the cone of critical directions, and a fundamental integral Weierstrass inequality involving a function whose role is parallel to the Hamiltonian of the problem. Given an admissible process, its set of active indices of the inequality restrictions has to be piecewise constant on the underlying time interval, the Lagrange multipliers associated with the inequality mixed constraints must be nonnegative and in fact they have to be zero whenever the corresponding index is inactive. The optimal control of the proposed optimal process need not be continuous but only measurable, see, for example, [1-17], where the authors study several optimal control problems having a degree of generality very similar to the one treated in this paper and where the continuity of the optimal controls is a crucial assumption in those sufficiency theories. In the first of the sufficiency theorems of this work, the deviation between optimal costs and feasible costs is estimated by quadratic functionals, two of them playing the role of the square of the norm of the classical Banach space $L^{1}$.

It is worth mentioning that second order sufficient conditions, as pointed out in [15], are necessary in nonlinear problems when the extremal is not unique or when an existence theorem is not applicable. In addition, the sufficient treatments have shown to be of crucial relevance in some parametric optimal control problems studying the analysis of stability or sensitivity, see, for example, [16,17]. In the previous references, the initial or final states are free, but they are restricted to lie in some surfaces delimited by curves; in contrast, the initial and final states of the nonparametric optimal control problem studied in this article are completely free, in the sense that they are not necessarily restricted to a parametrization, but they only must belong to any sets belonging to the images of a surface determined by a $C^{2}$ function. On the other hand, it is worth observing that all the crucial hypotheses of the 
sufficiency treatment studied in this article, are stated in the theorems, in contrast with other second order necessary and sufficiency theories that depend upon the verifiability of some crucial preliminary assumptions, see, for example, [18-20], where the necessary second order conditions for optimality depend on some previous hypotheses involving some notions of normality or regularity of a solution; or [11], where the corresponding sufficiency theory depends on the linear independence of some vectors whose role is the gradients of the active inequality and the equality restrictions. Finally, it is important to point out that, in [21,22], one can also find some sufficiency theories where the deviation between admissible and optimal costs around the optimal control has a quadratic growth.

The main novelties of this paper concern the facts that the sufficiency technique, used to prove Theorem 1, is independent of the standard hypothesis of continuity of the optimal controls, an assumption imposed in almost all the sufficiency theories having a similar degree of generality as the one studied in this article. In Corollary 1, the initial and final points of the states are not only variable, but they are completely free, in the sense that they may belong to any sets that must only be contained in a $C^{2}$ manifold, the sufficiency method employed to prove one of the results of the paper does not invoke classical sufficiency tools such as bounded matrix-valued Riccati equations, HamiltonJacobi inequalities, generalized notions of conjugate points, the linear independence of the gradients involving the active inequality and the equality constraints, insertions of the original optimal control problem in an abstract optimization problem involving a Banach space, or certain techniques based on arguments of convexity, see [1-17] for details. In the parametric sufficiency theorem of the article, if an admissible process satisfies all of its hypotheses, the former not only is a weak minimum, but the deviation between the optimal cost and the admissible costs is estimated by functionals playing similar roles of the squares of several norms.

The organization of the article is the following. In Section 2, we state a parametric optimal control problem we shall be concerned with together with some elementary definitions, and we also pose one of the main results of the paper. In Section 3, we establish a nonparametric optimal control problem we shall be interested in, some fundamental definitions, a corollary that forms one of the crucial results of the paper, and an example illustrating how one can apply the results of the article. Section 4 is dedicated to state three auxiliary results in which the proof of one of the theorems is based and whose proof is referred to [23]. Section 5 is dedicated to the proof of Theorem 1. Finally, in Section 6, some conclusions and some future directions of open problems are briefly enunciated.

\section{A Fundamental Theorem}

Suppose an interval $T:=\left[t_{0}, t_{1}\right]$ in $\mathbf{R}$ is given, in which we have functions $l: \mathbf{R}^{p} \rightarrow \mathbf{R}$, $\Psi_{i}: \mathbf{R}^{p} \rightarrow \mathbf{R}^{n}(i=0,1), L(t, x, u): T \times \mathbf{R}^{n} \times \mathbf{R}^{m} \rightarrow \mathbf{R}, f(t, x, u): T \times \mathbf{R}^{n} \times \mathbf{R}^{m} \rightarrow \mathbf{R}^{n}$, and $\varphi(t, x, u): T \times \mathbf{R}^{n} \times \mathbf{R}^{m} \rightarrow \mathbf{R}^{s}$. Set

$$
\mathcal{R}:=\left\{(t, x, u) \in T \times \mathbf{R}^{n} \times \mathbf{R}^{m} \mid \varphi_{\alpha}(t, x, u) \leq 0(\alpha \in R), \varphi_{\beta}(t, x, u)=0(\beta \in S)\right\}
$$

where $R:=\{1, \ldots, r\}$ and $S:=\{r+1, \ldots, s\}(r=0,1, \ldots, s)$. If $r=0$, then $R=\varnothing$ and we are not concerned with statements regarding $\varphi_{\alpha}$. Similarly, if $r=s$, then $S=\varnothing$, and we are not concerned with statements regarding $\varphi_{\beta}$.

Let $\left\{\Theta_{n}\right\}$ be a sequence of measurable functions and let $\Theta$ be a measurable function. We shall denote uniform convergence of $\left\{\Theta_{n}\right\}$ to $\Theta$ by $\Theta_{n} \stackrel{\mathrm{u}}{\longrightarrow} \Theta$. Similarly, strong convergence in $L^{p}$ by $\Theta_{n} \stackrel{L^{p}}{\longrightarrow} \Theta$ and weak convergence in $L^{p}$ by $\Theta_{n} \stackrel{L^{p}}{\longrightarrow} \Theta$.

We are going to assume throughout the article that $L, f$ and $\varphi=\left(\varphi_{1}, \ldots, \varphi_{s}\right)$ have first and second continuous derivatives with respect to $x$ and $u$ on $T \times \mathbf{R}^{n} \times \mathbf{R}^{m}$. Moreover, we shall suppose that the functions $l$ and $\Psi_{i}(i=0,1)$ are of class $C^{2}$ on $\mathbf{R}^{p}$.

Let $\mathcal{X}$ be the space of all absolutely continuous functions mapping $T$ to $\mathbf{R}^{n}$ and $\mathcal{U}_{\eta}:=L^{\infty}\left(T ; \mathbf{R}^{\eta}\right)(\eta \in \mathbf{N})$. 
Define $\mathcal{A}:=\mathcal{X} \times \mathcal{U}_{m} \times \mathbf{R}^{p}$, and keep in mind that the notation $z_{b}$ means any member $z_{b}:=(z, b)=(x, u, b) \in \mathcal{A}$. The parametric optimal control problem we shall be concerned with, denoted by $(\mathrm{P})$, consists of minimizing a functional of the form

$$
I\left(z_{b}\right):=l(b)+\int_{t_{0}}^{t_{1}} L(t, x(t), u(t)) d t
$$

over all $z_{b} \in \mathcal{A}$ satisfying the constraints

$$
\left\{\begin{array}{l}
\dot{x}(t)=f(t, x(t), u(t))(\text { a.e. in } T) \\
x\left(t_{i}\right)=\Psi_{i}(b) \text { for } i=0,1 \\
(t, x(t), u(t)) \in \mathcal{R}(t \in T)
\end{array}\right.
$$

The elements $b=\left(b_{1}, \ldots, b_{p}\right)^{*} \in \mathbf{R}^{p}$ (* means transpose) are called parameters, the members $z_{b}$ in $\mathcal{A}$ are called processes, and a process $z_{b}$ is feasible or admissible if it satisfies the constraints. The notation $z_{0 b_{0}}$ means a member $\left(z_{0}, b_{0}\right)=\left(x_{0}, u_{0}, b_{0}\right) \in \mathcal{A}$.

The following notation will allow us to introduce the main results of this section.

- A process $z_{0 b_{0}}$ is a solution of $(P)$ if it is feasible and $I\left(z_{0 b_{0}}\right) \leq I\left(z_{b}\right)$ for all feasible processes $z_{b}$. A feasible process $z_{0 b_{0}}$ is a weak minimum of $(\mathrm{P})$ if it is a minimum of $I$ with respect to the norm

$$
\left\|z_{b}\right\|:=|b|+\|(x, u)\|_{\infty}
$$

that is, if, for some $\epsilon>0, I\left(z_{0 b_{0}}\right) \leq I\left(z_{b}\right)$ for all feasible processes $z_{b}$ satisfying $\| z_{b}-$ $z_{0 b_{0}} \|<\epsilon$. In other words, if $I$ affords a weak minimum at $z_{0 b_{0}}$, then, if $z_{b}$ is admissible and it is sufficiently close to $z_{0 b_{0}}$, in the sense that the quantities $\left\|x-x_{0}\right\|_{\infty},\left\|u-u_{0}\right\|_{\infty}$ and $\left|b-b_{0}\right|$ are sufficiently small, then $I\left(z_{b}\right) \geq I\left(z_{0 b_{0}}\right)$. lem by

- For all $(t, x, u, \rho, \mu) \in T \times \mathbf{R}^{n} \times \mathbf{R}^{m} \times \mathbf{R}^{n} \times \mathbf{R}^{s}$, define the Hamiltonian of the prob-

$$
H(t, x, u, \rho, \mu):=\rho^{*} f(t, x, u)-L(t, x, u)-\mu^{*} \varphi(t, x, u) .
$$

Given $\rho \in \mathcal{X}$ and $\mu \in \mathcal{U}_{s}$ set, for all $(t, x, u) \in T \times \mathbf{R}^{n} \times \mathbf{R}^{m}$,

$$
F(t, x, u):=-H(t, x, u, \rho(t), \mu(t))-\dot{\rho}^{*}(t) x
$$

and let

$$
J\left(z_{b}\right):=\rho^{*}\left(t_{1}\right) x\left(t_{1}\right)-\rho^{*}\left(t_{0}\right) x\left(t_{0}\right)+l(b)+\int_{t_{0}}^{t_{1}} F(t, x(t), u(t)) d t .
$$

- The second variation of $J$ along $z_{b}$ in the direction $w_{\beta}$, is given by

$$
J^{\prime \prime}\left(z_{b} ; w_{\beta}\right):=\beta^{*} l^{\prime \prime}(b) \beta+\int_{t_{0}}^{t_{1}} 2 \Omega(t, x(t), u(t) ; t, y(t), v(t)) d t,
$$

where for all $(t, y, v) \in T \times \mathbf{R}^{n} \times \mathbf{R}^{m}$,

$$
2 \Omega(t, x(t), u(t) ; t, y, v):=y^{*} F_{x x}(t, x(t), u(t)) y+2 y^{*} F_{x u}(t, x(t), u(t)) v+v^{*} F_{u u}(t, x(t), u(t)) v,
$$

and the notation $w_{\beta}$ refers to any element $(y, v, \beta)$ in $\mathcal{X} \times L^{2}\left(T ; \mathbf{R}^{m}\right) \times \mathbf{R}^{p}$. In addition, $l^{\prime \prime}(b)$ is the second derivative of $l$ evaluated at $b$.

- Define

$$
E(t, x, u, v):=F(t, x, v)-F(t, x, u)-F_{u}(t, x, u)(v-u)
$$

- Given $x \in \mathcal{X}$ and $u \in L^{1}\left(T ; \mathbf{R}^{m}\right)$, set

$$
\mathcal{Q}(x, u):=\max \left\{\mathcal{Q}_{1}(x), \mathcal{Q}_{2}(u)\right\}
$$


where

$$
\mathcal{Q}_{1}(x):=\int_{t_{0}}^{t_{1}} V(\dot{x}(t)) d t, \quad \mathcal{Q}_{2}(u):=\int_{t_{0}}^{t_{1}} V(u(t)) d t, \quad V(e):=\left(1+|e|^{2}\right)^{1 / 2}-1 .
$$

- For all $x \in \mathcal{X}$ and all $u \in L^{1}\left(T ; \mathbf{R}^{m}\right)$, define

$$
D(x, u):=\max \left\{D_{1}(x), D_{2}(x, u)\right\}
$$

where

$$
D_{1}(x):=V\left(x\left(t_{0}\right)\right)+\mathcal{Q}_{1}(x) \text { and } D_{2}(x, u):=V\left(x\left(t_{0}\right)\right)+\mathcal{Q}_{2}(u) .
$$

Finally, given $(t, x, u) \in T \times \mathbf{R}^{n} \times \mathbf{R}^{m}$, denote by

$$
\mathcal{I}_{a}(t, x, u):=\left\{\alpha \in R \mid \varphi_{\alpha}(t, x, u)=0\right\},
$$

the set of active indices of $(t, x, u)$ with respect to the inequality restrictions. For all $z_{b} \in \mathcal{A}$, let $\mathcal{Y}\left(z_{b}\right)$ be the cone of all $w_{\beta} \in \mathcal{X} \times L^{2}\left(T ; \mathbf{R}^{m}\right) \times \mathbf{R}^{p}$ verifying

$$
\left\{\begin{array}{l}
\left.\dot{y}(t)=f_{x}(t, x(t), u(t)) y(t)+f_{u}(t, x(t), u(t)) v(t) \text { (a.e. in } T\right), y\left(t_{i}\right)=\Psi_{i}^{\prime}(b) \beta(i=0,1) . \\
\left.\varphi_{\alpha x}(t, x(t), u(t)) y(t)+\varphi_{\alpha u}(t, x(t), u(t)) v(t) \leq 0 \text { (a.e. in } T, \alpha \in \mathcal{I}_{a}(t, x(t), u(t))\right) . \\
\varphi_{\beta x}(t, x(t), u(t)) y(t)+\varphi_{\beta u}(t, x(t), u(t)) v(t)=0(\text { a.e. in } T, \beta \in S) .
\end{array}\right.
$$

The set $\mathcal{Y}\left(z_{b}\right)$ is the cone of critical directions with respect to $z_{b}$, and the symbol $\Psi_{i}^{\prime}$ $(i=0,1)$ means the derivative of $\Psi_{i}(i=0,1)$.

Theorem 1 below is a crucial tool in order to obtain Corollary 1 , the latter being the main result of the article. Theorem 1 gives sufficiency for weak minima of problem (P). Hypothesis (i) of Theorem 1 is the transversality condition, hypothesis (ii) is an inequality relation that was found during the original proof of Theorem 1, hypothesis (iii) is a modified version of the Legendre-Clebsch condition, hypothesis (iv) is the positivity of a quadratic integral on the cone of critical directions, and hypothesis (v) involves a Weierstrass integral inequality hypothesis. A remarkable component of Theorem 1 concerns the fact that the optimal control is not necessarily continuous but only measurable. The notation, $\Psi_{i}^{\prime \prime}\left(b_{0} ; \beta\right)$ $(i=0,1)$ is the second derivative of $\Psi_{i}(i=0,1)$ along $b_{0}$ in the direction $\beta$.

Theorem 1. Let $z_{0 b_{0}}$ be a feasible process. Suppose that $\mathcal{I}_{a}\left(\cdot, x_{0}(\cdot), u_{0}(\cdot)\right)$ is piecewise constant on $T$, that there exist $\rho \in \mathcal{X}, \mu \in \mathcal{U}_{s}$ with $\mu_{\alpha}(t) \geq 0, \mu_{\alpha}(t) \varphi_{\alpha}\left(t, x_{0}(t), u_{0}(t)\right)=0(\alpha \in R, t \in T)$ and $\delta, \epsilon>0$, such that

$$
\begin{gathered}
\dot{\rho}(t)=-H_{x}^{*}\left(t, x_{0}(t), u_{0}(t), \rho(t), \mu(t)\right)(\text { a.e. in } T), \\
H_{u}^{*}\left(t, x_{0}(t), u_{0}(t), \rho(t), \mu(t)\right)=0(t \in T),
\end{gathered}
$$

and the following is verified

(i) $l^{\prime *}\left(b_{0}\right)+\Psi_{1}^{\prime *}\left(b_{0}\right) \rho\left(t_{1}\right)-\Psi_{0}^{\prime *}\left(b_{0}\right) \rho\left(t_{0}\right)=0$.

(ii) $\rho^{*}\left(t_{1}\right) \Psi_{1}^{\prime \prime}\left(b_{0} ; \beta\right)-\rho^{*}\left(t_{0}\right) \Psi_{0}^{\prime \prime}\left(b_{0} ; \beta\right) \geq 0$ for all $\beta \in \mathbf{R}^{p}$.

(iii) $H_{u u}\left(t, x_{0}(t), u_{0}(t), \rho(t), \mu(t)\right) \leq 0($ a.e. in $T)$.

(iv) $J^{\prime \prime}\left(z_{0 b_{0}} ; w_{\beta}\right)>0$ for all $w_{\beta} \in \mathcal{Y}\left(z_{0 b_{0}}\right), w_{\beta} \not \equiv(0,0,0)$.

(v) For all $z_{b}$ feasible with $\left\|(x, u)-\left(x_{0}, u_{0}\right)\right\|_{\infty}<\epsilon, \int_{t_{0}}^{t_{1}} E\left(t, x(t), u_{0}(t), u(t)\right) d t \geq$ $\delta \mathcal{Q}\left(z-z_{0}\right)$.

Then, for some $\theta_{1}, \theta_{2}>0$ and all admissible processes $z_{b}$ satisfying $\left\|z_{b}-z_{0 b_{0}}\right\|<\theta_{1}$,

$$
I\left(z_{b}\right) \geq I\left(z_{0 b_{0}}\right)+\theta_{2} \min \left\{\left|b-b_{0}\right|^{2}, D\left(z-z_{0}\right)\right\} .
$$


In particular, $z_{0 b_{0}}$ is a weak minimum of $(P)$.

\section{The Main Result}

Suppose that we have an interval $T:=\left[t_{0}, t_{1}\right]$ in $\mathbf{R}$, two sets $\mathcal{B}_{0}, \mathcal{B}_{1} \subset \mathbf{R}^{n}$ and functions $\ell\left(a_{1}, a_{2}\right): \mathbf{R}^{n} \times \mathbf{R}^{n} \rightarrow \mathbf{R}, \mathcal{L}(t, x, u): T \times \mathbf{R}^{n} \times \mathbf{R}^{m} \rightarrow \mathbf{R}, g(t, x, u): T \times \mathbf{R}^{n} \times \mathbf{R}^{m} \rightarrow \mathbf{R}^{n}$ and $\phi(t, x, u): T \times \mathbf{R}^{n} \times \mathbf{R}^{m} \rightarrow \mathbf{R}^{s}$. Set

$$
\overline{\mathcal{R}}:=\left\{(t, x, u) \in T \times \mathbf{R}^{n} \times \mathbf{R}^{m} \mid \phi_{\alpha}(t, x, u) \leq 0(\alpha \in R), \phi_{\beta}(t, x, u)=0(\beta \in S)\right\}
$$

where $R:=\{1, \ldots, r\}$ and $S:=\{r+1, \ldots, s\}(r=0,1, \ldots, s)$. If $r=0$, then $R=\varnothing$ and we are not concerned with statements regarding $\phi_{\alpha}$. Similarly, if $r=s$, then $S=\varnothing$ and we are not concerned with statements regarding $\phi_{\beta}$.

We are going to assume throughout this section that $\mathcal{L}, g$ and $\phi=\left(\phi_{1}, \ldots, \phi_{s}\right)$ have first and second continuous derivatives with respect to $x$ and $u$ on $T \times \mathbf{R}^{n} \times \mathbf{R}^{m}$. Additionally, we suppose that the function $\ell$, is of class $C^{2}$ on $\mathbf{R}^{n} \times \mathbf{R}^{n}$.

Set $A:=\mathcal{X} \times \mathcal{U}_{m}$, where as usual, $\mathcal{X}$ denotes the space of absolutely continuous functions mapping $T$ to $\mathbf{R}^{n}$ and $\mathcal{U}_{m}:=L^{\infty}\left(T ; \mathbf{R}^{m}\right)$.

In this section, we shall be concerned with the nonparametric optimal control problem, denoted by $(\bar{P})$, of minimizing the functional

$$
\mathcal{J}(x, u):=\ell\left(x\left(t_{0}\right), x\left(t_{1}\right)\right)+\int_{t_{0}}^{t_{1}} \mathcal{L}(t, x(t), u(t)) d t
$$

over all pairs $(x, u) \in A$ satisfying the restrictions

$$
\left\{\begin{array}{l}
\dot{x}(t)=g(t, x(t), u(t))(\text { a.e. in } T) . \\
x\left(t_{i}\right) \in \mathcal{B}_{i} \text { for } i=0,1 . \\
(t, x(t), u(t)) \in \overline{\mathcal{R}}(t \in T) .
\end{array}\right.
$$

Members $(x, u)$ in $A$ are called processes, and a process $(x, u)$ is feasible if it satisfies the restrictions.

A process $\left(x_{0}, u_{0}\right)$ solves $(\bar{P})$ if it is feasible and $\mathcal{J}\left(x_{0}, u_{0}\right) \leq \mathcal{J}(x, u)$ for all feasible processes $(x, u)$. A feasible process $\left(x_{0}, u_{0}\right)$ is a weak minimum of $(\bar{P})$ if it is a minimum of $\mathcal{J}$ relative to the essential supremum norm, that is, if for some $\epsilon>0, \mathcal{J}\left(x_{0}, u_{0}\right) \leq \mathcal{J}(x, u)$ for all feasible processes satisfying $\left\|(x, u)-\left(x_{0}, u_{0}\right)\right\|_{\infty}<\epsilon$.

Let $\Psi: \mathbf{R}^{n} \rightarrow \mathbf{R}^{n} \times \mathbf{R}^{n}$ be any function of class $C^{2}$ such that $\mathcal{B}_{0} \times \mathcal{B}_{1} \subset \Psi\left(\mathbf{R}^{n}\right)$. Relate the nonparametric optimal control problem $(\bar{P})$ with the parametric optimal control problem given in Section 2, denoted by $\left(P_{\Psi}\right)$, that is, $\left(P_{\Psi}\right)$ is the parametric problem defined in Section 2, with the following data; $p=n, l=\ell \circ \Psi, L=\mathcal{L}, f=g, \varphi=\phi$ and $\Psi_{0}, \Psi_{1}$ the components of $\Psi$, that is, $\Psi=\left(\Psi_{0}, \Psi_{1}\right)$.

Lemma 1. The following is verified:

(i) $z_{b}$ is a feasible process of $\left(P_{\Psi}\right)$ if and only if $z=(x, u)$ is a feasible process of $(\bar{P})$ and $b \in \Psi^{-1}\left(x\left(t_{0}\right), x\left(t_{1}\right)\right)$.

(ii) If $z_{b}$ is a feasible process of $\left(P_{\Psi}\right)$, then

$$
\mathcal{J}(x, u)=I\left(z_{b}\right)
$$

(iii) If $z_{0 b_{0}}$ solves $\left(P_{\Psi}\right)$, then $\left(x_{0}, u_{0}\right)$ solves $(\bar{P})$.

Proof. This is precisely Lemma 1 of [23].

Corollary 1 below is an immediate consequence of Theorem 1 and Lemma 1. It gives sufficiency conditions of problem $(\bar{P})$. Once again, it is worthwhile observing that the optimal control is not necessarily continuous but only measurable. 
Corollary 1. Let $\Psi: \mathbf{R}^{n} \rightarrow \mathbf{R}^{n} \times \mathbf{R}^{n}$ be any function of class $C^{2}$ such that $\mathcal{B}_{0} \times \mathcal{B}_{1} \subset \Psi\left(\mathbf{R}^{n}\right)$ and let $\left(P_{\Psi}\right)$ be the parametric optimal control problem posed before enunciating Lemma 1. Let $z_{0 b_{0}}$ be a feasible process of $\left(P_{\Psi}\right)$. Suppose that $\mathcal{I}_{a}\left(\cdot, x_{0}(\cdot), u_{0}(\cdot)\right)$ is piecewise constant on $T$, there exist $\rho \in \mathcal{X}, \mu \in \mathcal{U}_{s}$ with $\mu_{\alpha}(t) \geq 0$, and $\mu_{\alpha}(t) \varphi_{\alpha}\left(t, x_{0}(t), u_{0}(t)\right)=0(\alpha \in R, t \in T), \delta, \epsilon>0$, such that

$$
\begin{gathered}
\dot{\rho}(t)=-H_{x}^{*}\left(t, x_{0}(t), u_{0}(t), \rho(t), \mu(t)\right)(\text { a.e. in } T), \\
H_{u}^{*}\left(t, x_{0}(t), u_{0}(t), \rho(t), \mu(t)\right)=0(t \in T),
\end{gathered}
$$

and the following is verified:

(i) $l^{\prime *}\left(b_{0}\right)+\Psi_{1}^{\prime *}\left(b_{0}\right) \rho\left(t_{1}\right)-\Psi_{0}^{\prime *}\left(b_{0}\right) \rho\left(t_{0}\right)=0$.

(ii) $\rho^{*}\left(t_{1}\right) \Psi_{1}^{\prime \prime}\left(b_{0} ; \beta\right)-\rho^{*}\left(t_{0}\right) \Psi_{0}^{\prime \prime}\left(b_{0} ; \beta\right) \geq 0$ for all $\beta \in \mathbf{R}^{n}$.

(iii) $H_{u u}\left(t, x_{0}(t), u_{0}(t), \rho(t), \mu(t)\right) \leq 0$ (a.e. in $\left.T\right)$.

(iv) $J^{\prime \prime}\left(z_{0 b_{0}} ; w_{\beta}\right)>0$ for all $w_{\beta} \in \mathcal{Y}\left(z_{0 b_{0}}\right), w_{\beta} \not \equiv(0,0,0)$.

(v) For all $z_{b}$ feasible with $\left\|(x, u)-\left(x_{0}, u_{0}\right)\right\|_{\infty}<\epsilon, \int_{t_{0}}^{t_{1}} E\left(t, x(t), u_{0}(t), u(t)\right) d t \geq$ $\delta \mathcal{Q}\left(z-z_{0}\right)$.

Then, $\left(x_{0}, u_{0}\right)$ is a weak minimum of $(\bar{P})$.

Remark 1. It is worth observing that our sufficiency theory can also be applied to isoperimetric problems of Bolza with inequality and equality constraints.

In order to illustrate this fact for the nonparametric problem studied in this section, let $\ell_{\gamma}: \mathbf{R}^{n} \times \mathbf{R}^{n} \rightarrow \mathbf{R}(\gamma=1, \ldots, K)$ be functions of class $C^{2}$ in $\mathbf{R}^{n} \times \mathbf{R}^{n}$. In addition, let $\mathcal{L}_{\gamma}(t, x, u): T \times \mathbf{R}^{n} \times \mathbf{R}^{m} \rightarrow \mathbf{R}(\gamma=1, \ldots, K)$ be functions having first and second continuous derivatives with respect to $x$ and $u$ on $T \times \mathbf{R}^{n} \times \mathbf{R}^{m}$, and consider the isoperimetric nonparametric optimal control problem of minimizing

$$
\mathcal{J}(x, u):=\ell\left(x\left(t_{0}\right), x\left(t_{1}\right)\right)+\int_{t_{0}}^{t_{1}} \mathcal{L}(t, x(t), u(t)) d t
$$

subject to

$$
\left\{\begin{array}{l}
\dot{x}(t)=g(t, x(t), u(t))(\text { a.e. in } T) . \\
x\left(t_{i}\right) \in \mathcal{B}_{i} \text { for } i=0,1 . \\
\mathcal{J}_{i}(x, u):=\ell_{i}\left(x\left(t_{0}\right), x\left(t_{1}\right)\right)+\int_{t_{0}}^{t_{1}} \mathcal{L}_{i}(t, x(t), u(t)) d t \leq 0(i=1, \ldots, k) . \\
\mathcal{J}_{j}(x, u):=\ell_{j}\left(x\left(t_{0}\right), x\left(t_{1}\right)\right)+\int_{t_{0}}^{t_{1}} \mathcal{L}_{j}(t, x(t), u(t)) d t=0(j=k+1, \ldots, K) . \\
(t, x(t), u(t)) \in \mathcal{\mathcal { R }}(t \in T) .
\end{array}\right.
$$

Additionally, set $\tilde{\mathcal{L}}(t, x, w, u):=\mathcal{L}(t, x, u)$,

$$
\tilde{g}(t, x, w, u):=\left(\begin{array}{c}
g(t, x, u) \\
\left(t_{1}-t_{0}\right)^{-1} \ell_{1}\left(x\left(t_{0}\right), x\left(t_{1}\right)\right)+\mathcal{L}_{1}(t, x, u) \\
\vdots \\
\left(t_{1}-t_{0}\right)^{-1} \ell_{K}\left(x\left(t_{0}\right), x\left(t_{1}\right)\right)+\mathcal{L}_{K}(t, x, u)
\end{array}\right)
$$

$\overline{\mathcal{B}}_{0}:=\mathcal{B}_{0} \times \mathcal{C}_{0}, \overline{\mathcal{B}}_{1}:=\mathcal{B}_{1} \times \mathcal{C}_{1}$, where $\mathcal{C}_{1}:=(-\infty, 0] \times \cdots \times(-\infty, 0] \times\{0\} \times \cdots \times\{0\}$ and $\mathcal{C}_{0}:=\{0\} \times \cdots \times\{0\}$. Here, in $\mathcal{C}_{1}$, there are $k$ intervals $(-\infty, 0]$ and $K-k$ singletons $\{0\}$. In $\mathcal{C}_{0}$, there are $K$ singletons $\{0\}$. Remark 1 follows from the fact that the isoperimetric optimal control problem stated above is equivalent to the nonparametric optimal control problem of minimizing 


$$
\mathcal{J}(x, w, u):=\ell\left(x\left(t_{0}\right), x\left(t_{1}\right)\right)+\int_{t_{0}}^{t_{1}} \tilde{\mathcal{L}}(t, x(t), w(t), u(t)) d t
$$

subject to

$$
\left\{\begin{array}{l}
(\dot{x}(t), \dot{w}(t))=\tilde{g}(t, x(t), w(t), u(t))(\text { a.e. in } T) \\
\left(x\left(t_{i}\right), w\left(t_{i}\right)\right) \in \overline{\mathcal{B}}_{i} \text { for } i=0,1 . \\
(t, x(t), u(t)) \in \overline{\mathcal{R}}(t \in T) .
\end{array}\right.
$$

Example 1 below shows how Corollary 1 can be applied. In the former, an inequalityequality constrained optimal control problem is solved by verifying that the first order sufficiency conditions

$\dot{\rho}(t)=-H_{x}^{*}\left(t, x_{0}(t), u_{0}(t), \rho(t), \mu(t)\right)($ a.e. in $T), \quad H_{u}^{*}\left(t, x_{0}(t), u_{0}(t), \rho(t), \mu(t)\right)=0(t \in T)$,

are satisfied by an element $\left(x_{0}, u_{0}, \rho, \mu\right)$. In addition, $\left(x_{0}, u_{0}, \rho, \mu\right)$ satisfies hypotheses (i), (ii), (iii), (iv) and (v) of Corollary 1 , and hence it is a weak minimum of $(\bar{P})$.

Example 1. Consider the nonparametric optimal control problem $(\bar{P})$ of minimizing

$$
\mathcal{J}(x, u):=x_{1}^{2}(0)+x_{2}^{2}(0)+x_{1}^{2}(1)+x_{2}^{2}(1)+\int_{0}^{1}\{\exp (t u(t))+\sin x(t)\} d t
$$

over all $(x, u) \in A$ satisfying the constraints

$$
\left\{\begin{array}{l}
\left(\dot{x}_{1}(t), \dot{x}_{2}(t)\right)=\left(u(t), \exp \left(-x_{1}(t)\right)-\exp (t u(t))\right)(\text { a.e. in }[0,1]) \\
x(0) \in\{0\} \times\{0\}, x(1) \in(-\infty, 0] \times\{0\} \\
(t, x(t), u(t)) \in \overline{\mathcal{R}}(t \in[0,1])
\end{array}\right.
$$

where

$$
\begin{gathered}
\overline{\mathcal{R}}:=\left\{(t, x, u) \in[0,1] \times \mathbf{R}^{2} \times \mathbf{R} \mid u^{2}-x_{1}^{2}-\exp \left(-x_{1}\right)-x_{1}+1 \leq 0, x_{1}+t u=0\right\}, \\
A:=\mathcal{X} \times \mathcal{U}_{1}, \\
\mathcal{X}:=\left\{x:[0,1] \rightarrow \mathbf{R}^{2} \mid x \text { is absolutely continuous on }[0,1]\right\}, \\
\mathcal{U}_{1}:=\{u:[0,1] \rightarrow \mathbf{R} \mid u \text { is essentially bounded on }[0,1]\} .
\end{gathered}
$$

For this example, the data of the nonparametric problem are given by $T=[0,1]$, $n=2, m=1, r=1, s=2, \mathcal{B}_{0}=\{0\} \times\{0\}, \mathcal{B}_{1}=(-\infty, 0] \times\{0\}, \ell\left(a_{1}, a_{2}\right)=\left(a_{1}^{1}\right)^{2}+$ $\left(a_{1}^{2}\right)^{2}+\left(a_{2}^{1}\right)^{2}+\left(a_{2}^{2}\right)^{2}, \mathcal{L}(t, x, u)=\exp (t u)+\sin x, g(t, x, u)=\left(u, \exp \left(-x_{1}\right)-\exp (t u)\right)^{*}$, $\phi_{1}(t, x, u)=u^{2}-x_{1}^{2}-\exp \left(-x_{1}\right)-x_{1}+1$ and $\phi_{2}(t, x, u)=x_{1}+t u$.

It is straightforwardly verified that the functions $\mathcal{L}, g, \phi=\left(\phi_{1}, \phi_{2}\right)$ and their first and second derivatives with respect to $x$ and $u$ are continuous on $T \times \mathbf{R}^{2} \times \mathbf{R}$. Moreover, the function $\ell$ is $C^{2}$ in $\mathbf{R}^{2} \times \mathbf{R}^{2}$.

Additionally, it is evident that the process $z_{0}=\left(x_{0}, u_{0}\right) \equiv(0,0,0)$ is admissible of $(\bar{P})$. Let $\Psi: \mathbf{R}^{2} \rightarrow \mathbf{R}^{2} \times \mathbf{R}^{2}$ be defined by $\Psi(b):=\left(\left(0, b_{2}\right),\left(b_{1}, b_{2}\right)\right)$. Clearly, $\Psi$ is $C^{2}$ in $\mathbf{R}^{2}$ and $\mathcal{B}_{0} \times \mathcal{B}_{1} \subset \Psi\left(\mathbf{R}^{2}\right)$. The related parametric problem denoted by $\left(P_{\Psi}\right)$ has the following data; $p=2, l=\ell \circ \Psi, L=\mathcal{L}, f=g, \varphi=\phi$, and $\Psi_{0}, \Psi_{1}$ the components of $\Psi$, that is, $\Psi=\left(\Psi_{0}, \Psi_{1}\right)$ with $\Psi_{0}(b)=\left(0, b_{2}\right)^{*}$ and $\Psi_{1}(b)=\left(b_{1}, b_{2}\right)^{*}\left(b \in \mathbf{R}^{2}\right)$.

Note that, if we set $b_{0}=\left(b_{01}, b_{02}\right):=(0,0)$, then $z_{0 b_{0}}=\left(x_{0}, u_{0}, b_{0}\right) \equiv(0,0,0,0,0)$ is feasible for $\left(P_{\Psi}\right)$. In addition, clearly, $\mathcal{I}_{a}\left(\cdot, x_{0}(\cdot), u_{0}(\cdot)\right)=\{1\}$ is constant on $T$. Let $\rho=\left(\rho_{1}, \rho_{2}\right) \equiv(0,-1), \mu=\left(\mu_{1}, \mu_{2}\right) \equiv(1,0)$ and note that $(\rho, \mu) \in \mathcal{X} \times \mathcal{U}_{2}, \mu_{1}(t) \geq 0$ and $\mu_{1}(t) \varphi_{1}\left(t, x_{0}(t), u_{0}(t)\right)=0(t \in T)$. 
Now,

$$
\begin{aligned}
H(t, x, u, \rho, \mu)=\rho_{1} u+\rho_{2} \exp \left(-x_{1}\right) & -\rho_{2} \exp (t u)-\exp (t u)-\sin x_{1} \\
& -\mu_{1}\left[u^{2}-x_{1}^{2}-\exp \left(-x_{1}\right)-x_{1}+1\right]-\mu_{2}\left[x_{1}+t u\right]
\end{aligned}
$$

and note that

$$
\begin{gathered}
H_{u}(t, x, u, \rho, \mu)=\rho_{1}-t \rho_{2} \exp (t u)-t \exp (t u)-2 \mu_{1} u-t \mu_{2} \\
H_{x}(t, x, u, \rho, \mu)=\left(\begin{array}{c}
-\rho_{2} \exp \left(-x_{1}\right)-\cos x_{1}+2 \mu_{1} x_{1}-\mu_{1} \exp \left(-x_{1}\right)+\mu_{1}-\mu_{2} \\
0
\end{array}\right)^{*} .
\end{gathered}
$$

It is straightforwardly verified that, for all $t \in T$,

$$
\dot{\rho}(t)=-H_{x}^{*}\left(t, x_{0}(t), u_{0}(t), \rho(t), \mu(t)\right) \quad \text { and } \quad H_{u}\left(t, x_{0}(t), u_{0}(t), \rho(t), \mu(t)\right)=0
$$

and then $\left(x_{0}, u_{0}, \rho, \mu\right)$ satisfies the first order sufficiency hypotheses of Corollary 1 . As $\Psi_{0}(b)=\left(0, b_{2}\right)^{*}, \Psi_{1}(b)=\left(b_{1}, b_{2}\right)^{*}, l(b)=b_{1}^{2}+2 b_{2}^{2}\left(b \in \mathbf{R}^{2}\right)$, then

$$
l^{\prime *}\left(b_{0}\right)+\Psi_{1}^{\prime *}\left(b_{0}\right) \rho(1)-\Psi_{0}^{\prime *}\left(b_{0}\right) \rho(0)=0
$$

and hence hypothesis (i) of Corollary 1 is satisfied. In addition, one can easily verify that,

$$
\rho^{*}(1) \Psi_{1}^{\prime \prime}\left(b_{0} ; \beta\right)-\rho^{*}(0) \Psi_{0}^{\prime \prime}\left(b_{0} ; \beta\right)=0 \text { for all } \beta \in \mathbf{R}^{2}
$$

and so hypothesis (ii) of Corollary 1 is fulfilled.

Now, for all $(t, x, u) \in T \times \mathbf{R}^{2} \times \mathbf{R}$,

$$
H(t, x, u, \rho(t), \mu(t))=-\sin x_{1}-u^{2}+x_{1}^{2}+x_{1}-1
$$

and so, for all $t \in T$,

$$
H_{u u}\left(t, x_{0}(t), u_{0}(t), \rho(t), \mu(t)\right)=-2 \leq 0
$$

which in turn implies that $\left(x_{0}, u_{0}, \rho, \mu\right)$ satisfies hypothesis (iii) of Corollary 1.

In addition, observe that, for all $t \in T$,

$$
\begin{gathered}
f_{x}\left(t, x_{0}(t), u_{0}(t)\right)=\left(\begin{array}{cc}
0 & 0 \\
-1 & 0
\end{array}\right), \quad f_{u}\left(t, x_{0}(t), u_{0}(t)\right)=\left(\begin{array}{c}
1 \\
-t
\end{array}\right), \\
\varphi_{1 x}\left(t, x_{0}(t), u_{0}(t)\right)=(0,0), \varphi_{1 u}\left(t, x_{0}(t), u_{0}(t)\right)=0, \varphi_{2 x}\left(t, x_{0}(t), u_{0}(t)\right)=(1,0), \varphi_{2 u}\left(t, x_{0}(t), u_{0}(t)\right)=t . \\
\text { Thus, } \mathcal{Y}\left(z_{0 b_{0}}\right) \text { is given by all } w_{\beta} \in \mathcal{X} \times L^{2}(T ; \mathbf{R}) \times \mathbf{R}^{2} \text { satisfying } \\
\left\{\begin{array}{l}
\left(\dot{y}_{1}(t), \dot{y}_{2}(t)\right)=\left(v(t),-y_{1}(t)-t v(t)\right)(\text { a.e. in } T), y(0)=\left(0, \beta_{2}\right)^{*}, y(1)=\left(\beta_{1}, \beta_{2}\right)^{*} . \\
y_{1}(t)+t v(t)=0(\text { a.e. in } T) .
\end{array}\right.
\end{gathered}
$$

Moreover, note that, for all $(t, x, u) \in T \times \mathbf{R}^{2} \times \mathbf{R}$,

$$
F(t, x, u)=\sin x_{1}+u^{2}-x_{1}^{2}-x_{1}+1
$$

and, for all $t \in T$,

$$
F_{x x}\left(t, x_{0}(t), u_{0}(t)\right)=\left(\begin{array}{cc}
-2 & 0 \\
0 & 0
\end{array}\right), \quad F_{x u}\left(t, x_{0}(t), u_{0}(t)\right)=(0,0), \quad F_{u u}\left(t, x_{0}(t), u_{0}(t)\right)=2 .
$$


Therefore, for all $w_{\beta} \in \mathcal{Y}\left(z_{0 b_{0}}\right)$,

$$
\frac{1}{2} J^{\prime \prime}\left(z_{0 b_{0}} ; w_{\beta}\right)=\beta_{1}^{2}+2 \beta_{2}^{2}+\int_{0}^{1}\left\{v^{2}(t)-y_{1}^{2}(t)\right\} d t=\beta_{1}^{2}+2 \beta_{2}^{2}+\int_{0}^{1}\left\{\dot{y}_{1}^{2}(t)-y_{1}^{2}(t)\right\} d t .
$$

Consequently,

$$
J^{\prime \prime}\left(z_{0 b_{0}} ; w_{\beta}\right)>0
$$

for all $w_{\beta} \in \mathcal{Y}\left(z_{0 b_{0}}\right), w_{\beta} \not \equiv(0,0,0)$, and so hypothesis (iv) of Corollary 1 is satisfied.

Now, observe that, if $z_{b}$ is feasible, for all $t \in T$,

$$
E\left(t, x(t), u_{0}(t), u(t)\right)=u^{2}(t) .
$$

Therefore, if $z_{b}$ is feasible,

$$
\begin{aligned}
& \int_{0}^{1} E\left(t, x(t), u_{0}(t), u(t)\right) d t=\int_{0}^{1} u^{2}(t) d t=\int_{0}^{1}\left\{u^{2}(t)+\left(\exp \left(-x_{1}(t)\right)-\exp (t u(t))\right)^{2}\right\} d t \\
= & \int_{0}^{1}\left\{\dot{x}_{1}^{2}(t)+\dot{x}_{2}^{2}(t)\right\} d t=\int_{0}^{1}|\dot{x}(t)|^{2} d t \geq \int_{0}^{1} V(\dot{x}(t)) d t=\mathcal{Q}_{1}\left(x-x_{0}\right) .
\end{aligned}
$$

In addition, if $z_{b}$ is feasible,

$$
\int_{0}^{1} E\left(t, x(t), u_{0}(t), u(t)\right) d t=\int_{0}^{1} u^{2}(t) d t \geq \int_{0}^{1} V(u(t)) d t=\mathcal{Q}_{2}\left(u-u_{0}\right) .
$$

Accordingly, for any $\epsilon>0$ and for any $z_{b}$ feasible with $\left\|(x, u)-\left(x_{0}, u_{0}\right)\right\|_{\infty}<\epsilon$,

$$
\int_{0}^{1} E\left(t, x(t), u_{0}(t), u(t)\right) d t \geq \max \left\{\mathcal{Q}_{1}\left(x-x_{0}\right), \mathcal{Q}_{2}\left(u-u_{0}\right)\right\}=\mathcal{Q}\left(x-x_{0}, u-u_{0}\right)=\mathcal{Q}\left(z-z_{0}\right) .
$$

Therefore, hypothesis (v) of Corollary 1 is fulfilled for any $\epsilon>0$ and $\delta=1$. By Corollary $1,\left(x_{0}, u_{0}\right)$ is a weak minimum of $(\bar{P})$.

Remark 2. The reader can find a concrete example concerning the existence of a purely measurable optimal control in which one of its components satisfies a classical type of amplitude constraints on the controls $u$.

Indeed, see Example 1 of [23], where one can find a concrete optimal control $u_{0}=$ $\left(u_{01}, u_{02}\right)$ with

$$
u_{02}(t):=\left\{\begin{array}{rrr}
1, & t=0 \\
1, & t \in \cup_{i=1}^{\infty}[1 /(2 i), 1 /(2 i-1)] \\
-1, & t \in \cup_{i=2}^{\infty}(1 /(2 i-1), 1 /(2 i-2))
\end{array}\right.
$$

and the feasible controls $u=\left(u_{1}, u_{2}\right)$ satisfying the amplitude constraints

$$
\left|u_{2}(t)-u_{02}(t)\right| \leq 1 \text { for all } t \in[0,1] .
$$

Remark 3. It would be of interest to see how the references quoted in this article or even the sufficiency theory presented in this paper can be generalized to the more complicated situation of the discrete-time case. See, for instance, [24], where time is measured in days in order to introduce a mathematical model to describe the outbreak of the Sars-Cov-2 in Ireland in March-May 2020. In the above reference, the optimal control treatment appeals to piecewise constant controls and state constraints for which a theoretical analysis is not amenable and hence a numerical approach is studied. It is worth mentioning that the optimal control model mentioned above saved lives and minimized the economical costs of the pharmaceutical interventions. 


\section{Auxiliary Lemmas}

Now, we state three auxiliary lemmas which are going to be useful in order to prove Theorem 1. The proof of these results are included in the proofs of Lemmas 2, 3, and 4 of [23], respectively. From now on, we are not going to relabel the subsequences of a given sequence since this fact will not modify our results.

Throughout this section, we shall assume that we are given an element $z_{0}:=\left(x_{0}, u_{0}\right) \in$ $\mathcal{X} \times L^{1}\left(T ; \mathbf{R}^{m}\right)$ and a sequence $\left\{z^{q}:=\left(x_{q}, u_{q}\right)\right\}$ in $\mathcal{X} \times L^{1}\left(T ; \mathbf{R}^{m}\right)$ such that

$$
\lim _{q \rightarrow \infty} D\left(z^{q}-z_{0}\right)=0 \quad \text { and } \quad d_{q}:=\left[2 D\left(z^{q}-z_{0}\right)\right]^{1 / 2}>0 \quad(q \in \mathbf{N}) .
$$

For all $q \in \mathbf{N}$, define

$$
y_{q}:=\frac{x_{q}-x_{0}}{d_{q}} \text { and } \quad v_{q}:=\frac{u_{q}-u_{0}}{d_{q}} .
$$

For all $q \in \mathbf{N}$, define

$$
W_{q}:=\max \left\{W_{1 q}, W_{2 q}\right\}
$$

where

$$
W_{1 q}:=\left[1+\frac{1}{2} V\left(\dot{x}_{q}-\dot{x}_{0}\right)\right]^{1 / 2}, \quad W_{2 q}:=\left[1+\frac{1}{2} V\left(u_{q}-u_{0}\right)\right]^{1 / 2} .
$$

Lemma 2. For some $v_{0} \in L^{2}\left(T ; \mathbf{R}^{m}\right)$ and some subsequence of $\left\{z^{q}\right\}, v_{q} \stackrel{L^{1}}{\rightarrow} v_{0}$ on $T$.

Lemma 3. There exist $\sigma_{0} \in L^{2}\left(T ; \mathbf{R}^{n}\right), \bar{y}_{0} \in \mathbf{R}^{n}$, and a subsequence of $\left\{z^{q}\right\}$, such that $\dot{y}_{q} \stackrel{L^{1}}{\longrightarrow} \sigma_{0}$ on $T$. Moreover, if for all $t \in T, y_{0}(t):=\bar{y}_{0}+\int_{t_{0}}^{t} \sigma_{0}(\tau) d \tau$, then $y_{q} \stackrel{u}{\longrightarrow} y_{0}$ on $T$.

Lemma 4. Suppose that $W_{q} \stackrel{L^{\infty}}{\longrightarrow} 1$ on $T$. Let $R_{q}, R_{0} \in L^{\infty}\left(T ; \mathbf{R}^{m \times m}\right)$, assume that $R_{q} \stackrel{L^{\infty}}{\longrightarrow} R_{0}$ on $T, R_{0}(t) \geq 0$ (a.e. in $T$ ), and let $v_{0}$ be as in Lemma 2. Then,

$$
\liminf _{q \rightarrow \infty} \int_{t_{0}}^{t_{1}} v_{q}^{*}(t) R_{q}(t) v_{q}(t) d t \geq \int_{t_{0}}^{t_{1}} v_{0}^{*}(t) R_{0}(t) v_{0}(t) d t .
$$

\section{Proof of Theorem 1}

The proof of Theorem 1 will be split up into two Lemmas. In Lemmas 5 and 6 below, we are assuming that all the hypotheses of Theorem 1 are satisfied. Before enunciating the lemmas, let us introduce some definitions.

First, note that given $x=\left(x_{1}, \ldots, x_{n}\right)^{*} \in \mathbf{R}^{n}$ and $b=\left(b_{1}, \ldots, b_{p}\right)^{*} \in \mathbf{R}^{p}$, if we define $x \mathbf{i}, b \mathbf{j} \in \mathbf{R}^{n+p}$ by $x \mathbf{i}:=\left(x_{1}, \ldots, x_{n}, 0, \ldots, 0\right)^{*}$ and $b \mathbf{j}:=\left(0, \ldots, 0, b_{1}, \ldots, b_{p}\right)^{*}$, then

$$
x \mathbf{i}+b \mathbf{j}=\left(x_{1}, \ldots, x_{n}, b_{1}, \ldots, b_{p}\right)^{*}=\left(\begin{array}{c}
x \\
b
\end{array}\right) \in \mathbf{R}^{n+p} .
$$

Define $\tilde{F}: T \times \mathbf{R}^{n+p} \times \mathbf{R}^{m} \rightarrow \mathbf{R}$ by

$$
\tilde{F}(t, \xi, u):=\frac{l\left(\xi_{n+1}, \ldots, \xi_{n+p}\right)}{t_{1}-t_{0}}+F\left(t, \xi_{1}, \ldots, \xi_{n}, u\right) .
$$

Note that the Weierstrass function $\tilde{E}: T \times \mathbf{R}^{n+p} \times \mathbf{R}^{m} \times \mathbf{R}^{m} \rightarrow \mathbf{R}$ of $\tilde{F}$ is defined by

$$
\tilde{E}(t, \xi, u, v):=\tilde{F}(t, \xi, v)-\tilde{F}(t, \xi, u)-\tilde{F}_{u}(t, \xi, u)(v-u) .
$$

As one readily verifies, for all $(t, x, u, v) \in T \times \mathbf{R}^{n} \times \mathbf{R}^{m} \times \mathbf{R}^{m}$ and all $b \in \mathbf{R}^{p}$,

$$
\tilde{E}(t, x \mathbf{i}+b \mathbf{j}, u, v)=E(t, x, u, v) .
$$




\section{Define}

$$
\tilde{J}\left(z_{b}\right):=\rho^{*}\left(t_{1}\right) x\left(t_{1}\right)-\rho^{*}\left(t_{0}\right) x\left(t_{0}\right)+\int_{t_{0}}^{t_{1}} \tilde{F}(t, x(t) \mathbf{i}+b \mathbf{j}, u(t)) d t .
$$

We have that $J\left(z_{b}\right)=\tilde{J}\left(z_{b}\right)$ for all $z_{b} \in \mathcal{A}$, and

$$
\tilde{J}\left(z_{b}\right)=\tilde{J}\left(z_{0 b_{0}}\right)+\tilde{J}^{\prime}\left(z_{0 b_{0}} ; z_{b}-z_{0 b_{0}}\right)+\tilde{K}\left(z_{0 b_{0}} ; z_{b}\right)+\tilde{\mathcal{E}}\left(z_{0 b_{0}} ; z_{b}\right)
$$

where

$$
\begin{aligned}
\tilde{\mathcal{E}}\left(z_{0 b_{0}} ; z_{b}\right) & :=\int_{t_{0}}^{t_{1}} \tilde{E}\left(t, x(t) \mathbf{i}+b \mathbf{j}, u_{0}(t), u(t)\right) d t, \\
\tilde{K}\left(z_{0 b_{0}} ; z_{b}\right):= & \int_{t_{0}}^{t_{1}}\left\{\tilde{M}(t, x(t) \mathbf{i}+b \mathbf{j})+\left[u^{*}(t)-u_{0}^{*}(t)\right] \tilde{N}(t, x(t) \mathbf{i}+b \mathbf{j})\right\} d t, \\
\tilde{J}^{\prime}\left(z_{0 b_{0}} ; z_{b}-z_{0 b_{0}}\right):= & \rho^{*}\left(t_{1}\right)\left[x\left(t_{1}\right)-x_{0}\left(t_{1}\right)\right]-\rho^{*}\left(t_{0}\right)\left[x\left(t_{0}\right)-x_{0}\left(t_{0}\right)\right] \\
& \quad+\int_{t_{0}}^{t_{1}}\left\{\tilde{F}_{\tilde{\zeta}}\left(t, x_{0}(t) \mathbf{i}+b_{0} \mathbf{j}, u_{0}(t)\right)\left(\left[x(t)-x_{0}(t)\right] \mathbf{i}+\left[b-b_{0}\right] \mathbf{j}\right)\right. \\
& \left.+\tilde{F}_{u}\left(t, x_{0}(t) \mathbf{i}+b_{0} \mathbf{j}, u_{0}(t)\right)\left(u(t)-u_{0}(t)\right)\right\} d t,
\end{aligned}
$$

and $\tilde{M}, \tilde{N}$ are given by

$$
\begin{array}{r}
\tilde{M}(t, x \mathbf{i}+b \mathbf{j}):=\tilde{F}\left(t, x \mathbf{i}+b \mathbf{j}, u_{0}(t)\right)-\tilde{F}\left(t, x_{0}(t) \mathbf{i}+b_{0} \mathbf{j}, u_{0}(t)\right) \\
-\tilde{F}_{\xi}\left(t, x_{0}(t) \mathbf{i}+b_{0} \mathbf{j}, u_{0}(t)\right)\left(\left[x-x_{0}(t)\right] \mathbf{i}+\left[b-b_{0}\right] \mathbf{j}\right), \\
\tilde{N}(t, x \mathbf{i}+b \mathbf{j}):=\tilde{F}_{u}^{*}\left(t, x \mathbf{i}+b \mathbf{j}, u_{0}(t)\right)-\tilde{F}_{u}^{*}\left(t, x_{0}(t) \mathbf{i}+b_{0} \mathbf{j}, u_{0}(t)\right) .
\end{array}
$$

We have

$$
\begin{aligned}
\tilde{M}(t, x \mathbf{i}+b \mathbf{j})= & \frac{1}{2}\left(\left[x^{*}-x_{0}^{*}(t)\right] \mathbf{i}+\left[b^{*}-b_{0}^{*}\right] \mathbf{j}\right) \tilde{P}(t, x \mathbf{i}+b \mathbf{j})\left(\left[x-x_{0}(t)\right] \mathbf{i}+\left[b-b_{0}\right] \mathbf{j}\right), \\
& \tilde{N}(t, x \mathbf{i}+b \mathbf{j})=\tilde{Q}(t, x \mathbf{i}+b \mathbf{j})\left(\left[x-x_{0}(t)\right] \mathbf{i}+\left[b-b_{0}\right] \mathbf{j}\right),
\end{aligned}
$$

where

$$
\begin{aligned}
\tilde{P}(t, x \mathbf{i}+b \mathbf{j}) & :=2 \int_{0}^{1}(1-\lambda) \tilde{F}_{\xi \xi}\left(t,\left[x_{0}(t)+\lambda\left(x-x_{0}(t)\right)\right] \mathbf{i}+\left[b_{0}+\lambda\left(b-b_{0}\right)\right] \mathbf{j}, u_{0}(t)\right) d \lambda, \\
\tilde{Q}(t, x \mathbf{i}+b \mathbf{j}) & :=\int_{0}^{1} \tilde{F}_{u \xi}\left(t,\left[x_{0}(t)+\lambda\left(x-x_{0}(t)\right)\right] \mathbf{i}+\left[b_{0}+\lambda\left(b-b_{0}\right)\right] \mathbf{j}, u_{0}(t)\right) d \lambda .
\end{aligned}
$$

Lemma 5. If the conclusion of Theorem 1 is false, then there exists a subsequence $\left\{z_{b_{q}}^{q}\right\}$ of feasible processes such that

$$
\lim _{q \rightarrow \infty} D\left(z^{q}-z_{0}\right)=0 \quad \text { and } \quad d_{q}:=\left[2 D\left(z^{q}-z_{0}\right)\right]^{1 / 2}>0 \quad(q \in \mathbf{N}) .
$$

Proof. If the conclusion of Theorem 1 is false, then for all $\theta_{1}, \theta_{2}>0$, there exists a feasible process $z_{b}$ such that

$$
\left\|z_{b}-z_{0 b_{0}}\right\|<\theta_{1} \quad \text { and } I\left(z_{b}\right)<I\left(z_{0 b_{0}}\right)+\theta_{2} \min \left\{\left|b-b_{0}\right|^{2}, D\left(z-z_{0}\right)\right\} .
$$

As

$$
\mu_{\alpha}(t) \geq 0(\alpha \in R \text {, a.e. in } T),
$$

if $z_{b}$ is feasible, then $I\left(z_{b}\right) \geq J\left(z_{b}\right)$. In addition, since

$$
\mu_{\alpha}(t) \varphi_{\alpha}\left(t, x_{0}(t), u_{0}(t)\right)=0(\alpha \in R \text {, a.e. in } T)
$$


then $I\left(z_{0 b_{0}}\right)=J\left(z_{0 b_{0}}\right)$. Therefore, (3) implies that, for all $\theta_{1}, \theta_{2}>0$, there exists $z_{b}$ feasible with

$$
\left\|z_{b}-z_{0 b_{0}}\right\|<\theta_{1} \quad \text { and } \quad J\left(z_{b}\right)<J\left(z_{0 b_{0}}\right)+\theta_{2} \min \left\{\left|b-b_{0}\right|^{2}, D\left(z-z_{0}\right)\right\} .
$$

Thus, if the conclusion of Theorem 1 is false, then, for all $q \in \mathbf{N}$, there exists $z_{b_{q}}^{q}$ feasible such that

$$
\left\|z_{b_{q}}^{q}-z_{0 b_{0}}\right\|<\min \{\epsilon, 1 / q\}, \quad J\left(z_{b_{q}}^{q}\right)-J\left(z_{0 b_{0}}\right)<\min \left\{\frac{\left|b_{q}-b_{0}\right|^{2}}{q}, \frac{D\left(z^{q}-z_{0}\right)}{q}\right\} .
$$

Clearly, the first relation in (4) implies that

$$
\lim _{q \rightarrow \infty} D\left(z^{q}-z_{0}\right)=0 .
$$

In addition, $D\left(z^{q}-z_{0}\right)=0$ if and only if $z^{q}=z_{0}$. Then, by the second relation of (4),

$$
D\left(z^{q}-z_{0}\right)=0 \Longrightarrow b_{q} \neq b_{0} .
$$

Suppose $D\left(z^{q}-z_{0}\right)=0$ for infinitely many $q^{\prime}$ s. For $i=0,1$, we have

$$
\begin{gathered}
0=x_{q}\left(t_{i}\right)-x_{0}\left(t_{i}\right)=\Psi_{i}\left(b_{q}\right)-\Psi_{i}\left(b_{0}\right)=\int_{0}^{1} \Psi_{i}^{\prime}\left(b_{0}+\lambda\left[b_{q}-b_{0}\right]\right)\left(b_{q}-b_{0}\right) d \lambda, \\
0=\Psi_{i}\left(b_{q}\right)-\Psi_{i}\left(b_{0}\right)=\Psi_{i}^{\prime}\left(b_{0}\right)\left(b_{q}-b_{0}\right)+\int_{0}^{1}(1-\lambda) \Psi_{i}^{\prime \prime}\left(b_{0}+\lambda\left[b_{q}-b_{0}\right] ; b_{q}-b_{0}\right) d \lambda .
\end{gathered}
$$

Denoting by $\left(b_{q}, b_{0}\right)$ the line segment in $\mathbf{R}^{p}$ joining the points $b_{q}$ and $b_{0}$, by the second relation of (4), by condition (i) of Theorem 1, by (6), and the mean value theorem, there exists $\Xi_{q} \in\left(b_{q}, b_{0}\right)$ such that

$$
\begin{aligned}
0 & >J\left(z_{0 b_{q}}\right)-J\left(z_{0 b_{0}}\right) \\
& =l\left(b_{q}\right)-l\left(b_{0}\right) \\
& =l^{\prime}\left(b_{0}\right)\left(b_{q}-b_{0}\right)+\frac{1}{2}\left(b_{q}-b_{0}\right)^{*} l^{\prime \prime}\left(\Xi_{q}\right)\left(b_{q}-b_{0}\right) \\
& =\rho^{*}\left(t_{0}\right) \Psi_{0}^{\prime}\left(b_{0}\right)\left(b_{q}-b_{0}\right)-\rho^{*}\left(t_{1}\right) \Psi_{1}^{\prime}\left(b_{0}\right)\left(b_{q}-b_{0}\right)+\frac{1}{2}\left(b_{q}-b_{0}\right)^{*} l^{\prime \prime}\left(\Xi_{q}\right)\left(b_{q}-b_{0}\right) \\
& =\sum_{i=0}^{1}(-1)^{i+1} \int_{0}^{1}(1-\lambda) \rho^{*}\left(t_{i}\right) \Psi_{i}^{\prime \prime}\left(b_{0}+\lambda\left[b_{q}-b_{0}\right] ; b_{q}-b_{0}\right) d \lambda+\frac{1}{2}\left(b_{q}-b_{0}\right)^{*} l^{\prime \prime}\left(\Xi_{q}\right)\left(b_{q}-b_{0}\right) .
\end{aligned}
$$

Choose an appropriate subsequence of $\left\{\left(b_{q}-b_{0}\right) /\left|b_{q}-b_{0}\right|\right\}$, such that

$$
\lim _{q \rightarrow \infty} \frac{b_{q}-b_{0}}{\left|b_{q}-b_{0}\right|}=\beta_{0}
$$

for some $\beta_{0} \in \mathbf{R}^{p}$ with $\left|\beta_{0}\right|=1$. By (5),

$$
\Psi_{i}^{\prime}\left(b_{0}\right) \beta_{0}=0 \quad(i=0,1) .
$$

By (7) and (8) and condition (ii) of Theorem 1, it follows that $0 \geq \frac{1}{2}\left[\rho^{*}\left(t_{1}\right) \Psi_{1}^{\prime \prime}\left(b_{0} ; \beta_{0}\right)-\rho^{*}\left(t_{0}\right) \Psi_{0}^{\prime \prime}\left(b_{0} ; \beta_{0}\right)+\beta_{0}^{*} l^{\prime \prime}\left(b_{0}\right) \beta_{0}\right] \geq \frac{1}{2} \beta_{0}^{*} l^{\prime \prime}\left(b_{0}\right) \beta_{0}=\frac{1}{2} J^{\prime \prime}\left(z_{0 b_{0}} ; 0_{\beta_{0}}\right)$ which contradicts (iv) of Theorem 1 . Therefore, we may assume that, for all $q \in \mathbf{N}$,

$$
d_{q}=\left[2 D\left(z^{q}-z_{0}\right)\right]^{1 / 2}>0 .
$$


Lemma 6. If the conclusion of Theorem 1 is false, then hypothesis (iv) of Theorem 1 is false.

Proof. Let $\left\{z_{b_{q}}^{q}\right\}$ be the sequence of feasible processes given in Lemma 5. Then,

$$
\lim _{q \rightarrow \infty} D\left(z^{q}-z_{0}\right)=0 \quad \text { and } \quad d_{q}=\left[2 D\left(z^{q}-z_{0}\right)\right]^{1 / 2}>0 \quad(q \in \mathbf{N}) .
$$

Case (1): First, suppose that the sequence $\left\{\left(b_{q}-b_{0}\right) / d_{q}\right\}$ is bounded in $\mathbf{R}^{p}$.

For all $q \in \mathbf{N}$, define

$$
y_{q}:=\frac{x_{q}-x_{0}}{d_{q}}, \quad v_{q}:=\frac{u_{q}-u_{0}}{d_{q}}, \quad \omega_{q}:=y_{q} \mathbf{i}+\frac{b_{q}-b_{0}}{d_{q}} \mathbf{j} .
$$

By Lemma 2, there exist $v_{0} \in L^{2}\left(T ; \mathbf{R}^{m}\right)$ and a subsequence of $\left\{z^{q}\right\}$, such that $v_{q} \stackrel{L^{1}}{\rightarrow} v_{0}$ on $T$. By Lemma 3 , there exist $\sigma_{0} \in L^{2}\left(T ; \mathbf{R}^{n}\right), \bar{y}_{0} \in \mathbf{R}^{n}$, and a subsequence of $\left\{z^{q}\right\}$, such that if $y_{0}(t):=\bar{y}_{0}+\int_{t_{0}}^{t} \sigma_{0}(\tau) d \tau(t \in T)$, then

$$
y_{q} \stackrel{\mathrm{u}}{\longrightarrow} y_{0} \text { on } T \text {. }
$$

Since the sequence $\left\{\left(b_{q}-b_{0}\right) / d_{q}\right\}$ is bounded in $\mathbf{R}^{p}$, then we may assume that there exists some $\beta_{0} \in \mathbf{R}^{p}$ such that

$$
\lim _{q \rightarrow \infty} \frac{b_{q}-b_{0}}{d_{q}}=\beta_{0} .
$$

First, we are going to show that for $i=0,1$,

$$
y_{0}\left(t_{i}\right)=\Psi_{i}^{\prime}\left(b_{0}\right) \beta_{0} .
$$

Observe that, for $i=0,1$ and all $q \in \mathbf{N}$, we have that

$$
y_{q}\left(t_{i}\right)=\int_{0}^{1} \Psi_{i}^{\prime}\left(b_{0}+\lambda\left[b_{q}-b_{0}\right]\right) \frac{\left(b_{q}-b_{0}\right)}{d_{q}} d \lambda
$$

By (9), (10) and (12), we obtain (11). Now, we claim that

$$
J^{\prime \prime}\left(z_{0 b_{0}} ; w_{0 \beta_{0}}\right) \leq 0, \quad w_{0 \beta_{0}}=\left(y_{0}, v_{0}, \beta_{0}\right) \not \equiv(0,0,0) .
$$

To prove it, observe that by (2), (9) and (10),

$$
\begin{aligned}
& \frac{\tilde{M}\left(\cdot, x_{q}(\cdot) \mathbf{i}+b_{q} \mathbf{j}\right)}{d_{q}^{2}}= \frac{1}{2} \omega_{q}^{*}(\cdot) \tilde{P}\left(\cdot, x_{q}(\cdot) \mathbf{i}+b_{q} \mathbf{j}\right) \omega_{q}(\cdot) \stackrel{L^{\infty}}{\longrightarrow} \\
& \frac{1}{2}\left[y_{0}^{*}(\cdot) \mathbf{i}+\beta_{0}^{*} \mathbf{j}\right] \tilde{F}_{\xi \xi}\left(\cdot, x_{0}(\cdot) \mathbf{i}+b_{0} \mathbf{j}, u_{0}(\cdot)\right)\left[y_{0}(\cdot) \mathbf{i}+\beta_{0} \mathbf{j}\right], \\
& \frac{\tilde{N}\left(\cdot, x_{q}(\cdot) \mathbf{i}+b_{q} \mathbf{j}\right)}{d_{q}}=\tilde{Q}\left(\cdot, x_{q}(\cdot) \mathbf{i}+b_{q} \mathbf{j}\right) \omega_{q}(\cdot) \stackrel{L^{\infty}}{\longrightarrow} \tilde{F}_{u \xi}\left(\cdot, x_{0}(\cdot) \mathbf{i}+b_{0} \mathbf{j}, u_{0}(\cdot)\right)\left[y_{0}(\cdot) \mathbf{i}+\beta_{0} \mathbf{j}\right]
\end{aligned}
$$

both on $T$. This fact, combined with Lemma 2, implies that

$$
\begin{array}{r}
\lim _{q \rightarrow \infty} \frac{\tilde{K}\left(z_{0 b_{0}} ; z_{b_{q}}^{q}\right)}{d_{q}^{2}}=\frac{1}{2} \int_{t_{0}}^{t_{1}}\left\{\left[y_{0}^{*}(t) \mathbf{i}+\beta_{0}^{*} \mathbf{j}\right] \tilde{F}_{\xi \tilde{\xi}}\left(t, x_{0}(t) \mathbf{i}+b_{0} \mathbf{j}, u_{0}(t)\right)\left[y_{0}(t) \mathbf{i}+\beta_{0} \mathbf{j}\right]\right. \\
\left.+2 v_{0}^{*}(t) \tilde{F}_{u \tilde{\xi}}\left(t, x_{0}(t) \mathbf{i}+b_{0} \mathbf{j}, u_{0}(t)\right)\left[y_{0}(t) \mathbf{i}+\beta_{0} \mathbf{j}\right]\right\} d t .
\end{array}
$$


Since $\left(x_{0}, u_{0}, \rho, \mu\right)$ satisfies the conditions

$\dot{\rho}(t)=-H_{x}^{*}\left(t, x_{0}(t), u_{0}(t), \rho(t), \mu(t)\right)($ a.e. in $T), \quad H_{u}^{*}\left(t, x_{0}(t), u_{0}(t), \rho(t), \mu(t)\right)=0(t \in T)$, and by hypothesis (i) of Theorem 1, we have that

$$
\begin{aligned}
& \lim _{q \rightarrow \infty} \frac{\tilde{J}^{\prime}\left(z_{0 b_{0}} ; z_{b_{q}}^{q}-z_{0 b_{0}}\right)}{d_{q}^{2}} \\
= & \lim _{q \rightarrow \infty} \frac{1}{d_{q}^{2}}\left[\rho^{*}\left(t_{1}\right)\left(x_{q}\left(t_{1}\right)-x_{0}\left(t_{1}\right)\right)-\rho^{*}\left(t_{0}\right)\left(x_{q}\left(t_{0}\right)-x_{0}\left(t_{0}\right)\right)+l^{\prime}\left(b_{0}\right)\left(b_{q}-b_{0}\right)\right] \\
= & \lim _{q \rightarrow \infty} \frac{1}{d_{q}^{2}}\left[\rho^{*}\left(t_{1}\right)\left(\Psi_{1}\left(b_{q}\right)-\Psi_{1}\left(b_{0}\right)-\Psi_{1}^{\prime}\left(b_{0}\right)\left(b_{q}-b_{0}\right)\right)-\rho^{*}\left(t_{0}\right)\left(\Psi_{0}\left(b_{q}\right)-\Psi_{0}\left(b_{0}\right)-\Psi_{0}^{\prime}\left(b_{0}\right)\left(b_{q}-b_{0}\right)\right)\right] \\
= & \lim _{q \rightarrow \infty} \frac{1}{d_{q}^{2}} \int_{0}^{1} \sum_{i=0}^{1}(-1)^{i+1}(1-\lambda) \rho^{*}\left(t_{i}\right) \Psi_{i}^{\prime \prime}\left(b_{0}+\lambda\left[b_{q}-b_{0}\right] ; b_{q}-b_{0}\right) d \lambda \\
= & \frac{1}{2}\left[\rho^{*}\left(t_{1}\right) \Psi_{1}^{\prime \prime}\left(b_{0} ; \beta_{0}\right)-\rho^{*}\left(t_{0}\right) \Psi_{0}^{\prime \prime}\left(b_{0} ; \beta_{0}\right)\right] .
\end{aligned}
$$

Consequently, by (1), the fact that

$$
J\left(z_{b_{q}}^{q}\right)-J\left(z_{0 b_{0}}\right)<\min \left\{\frac{\left|b_{q}-b_{0}\right|^{2}}{q}, \frac{D\left(z^{q}-z_{0}\right)}{q}\right\},
$$

(15) and condition (ii) of Theorem 1 ,

$$
0 \geq \lim _{q \rightarrow \infty} \frac{\tilde{K}\left(z_{0 b_{0}} ; z_{b_{q}}^{q}\right)}{d_{q}^{2}}+\liminf _{q \rightarrow \infty} \frac{\tilde{\mathcal{E}}\left(z_{0 b_{0}} ; z_{b_{q}}^{q}\right)}{d_{q}^{2}} .
$$

Now, for all $t \in T$ and $q \in \mathbf{N}$,

$$
\frac{1}{d_{q}^{2}} \tilde{E}\left(t, x_{q}(t) \mathbf{i}+b_{q} \mathbf{j}, u_{0}(t), u_{q}(t)\right)=\frac{1}{2} v_{q}^{*}(t) R_{q}(t) v_{q}(t),
$$

where

$$
R_{q}(t):=2 \int_{0}^{1}(1-\lambda) \tilde{F}_{u u}\left(t, x_{q}(t) \mathbf{i}+b_{q} \mathbf{j}, u_{0}(t)+\lambda\left[u_{q}(t)-u_{0}(t)\right]\right) d \lambda .
$$

As one readily verifies,

$$
R_{q}(\cdot) \stackrel{L^{\infty}}{\longrightarrow} R_{0}(\cdot):=\tilde{F}_{u u}\left(\cdot, x_{0}(\cdot) \mathbf{i}+b_{0} \mathbf{j}, u_{0}(\cdot)\right) \text { on } T .
$$

By hypothesis (iii) of Theorem 1, we have

$$
\tilde{F}_{u u}\left(t, x_{0}(t) \mathbf{i}+b_{0} \mathbf{j}, u_{0}(t)\right)=R_{0}(t) \geq 0 \quad(\text { a.e. in } T) .
$$

For all $q \in \mathbf{N}$, define

$$
W_{q}:=\max \left\{W_{1 q}, W_{2 q}\right\}
$$

where

$$
W_{1 q}:=\left[1+\frac{1}{2} V\left(\dot{x}_{q}-\dot{x}_{0}\right)\right]^{1 / 2}, \quad W_{2 q}:=\left[1+\frac{1}{2} V\left(u_{q}-u_{0}\right)\right]^{1 / 2} .
$$

By the fact that

$$
\left\|z_{b_{q}}^{q}-z_{0 b_{0}}\right\|<\frac{1}{q}
$$


and the admissibility of $z_{b_{q},}^{q} W_{q} \stackrel{L^{\infty}}{\longrightarrow} 1$ on $T$. With this in mind, by (17) and Lemma 4,

$$
\begin{aligned}
\liminf _{q \rightarrow \infty} \frac{\tilde{\mathcal{E}}\left(z_{0 b_{0}} ; z_{b_{q}}^{q}\right)}{d_{q}^{2}} & =\liminf _{q \rightarrow \infty} \frac{1}{d_{q}^{2}} \int_{t_{0}}^{t_{1}} \tilde{E}\left(t, x_{q}(t) \mathbf{i}+b_{q} \mathbf{j}, u_{0}(t), u_{q}(t)\right) d t \\
& =\frac{1}{2} \liminf _{q \rightarrow \infty} \int_{t_{0}}^{t_{1}} v_{q}^{*}(t) R_{q}(t) v_{q}(t) d t \geq \frac{1}{2} \int_{t_{0}}^{t_{1}} v_{0}^{*}(t) R_{0}(t) v_{0}(t) d t .
\end{aligned}
$$

By (16) and (18), we have

$$
\begin{aligned}
0 & \int_{t_{0}}^{t_{1}}\left\{v_{0}^{*}(t) \tilde{F}_{u u}\left(t, x_{0}(t) \mathbf{i}+b_{0} \mathbf{j}, u_{0}(t)\right) v_{0}(t)+2 v_{0}^{*}(t) \tilde{F}_{u \xi}\left(t, x_{0}(t) \mathbf{i}+b_{0} \mathbf{j}, u_{0}(t)\right)\left[y_{0}(t) \mathbf{i}+\beta_{0} \mathbf{j}\right]\right. \\
& \left.+\left[y_{0}^{*}(t) \mathbf{i}+\beta_{0}^{*} \mathbf{j}\right] \tilde{F}_{\xi \xi}\left(t, x_{0}(t) \mathbf{i}+b_{0} \mathbf{j}, u_{0}(t)\right)\left[y_{0}(t) \mathbf{i}+\beta_{0} \mathbf{j}\right]\right\} d t
\end{aligned}
$$

Now, let us show that $w_{0 \beta_{0}} \not \equiv(0,0,0)$. By (16), condition (v) of Theorem 1 and the fact that $V(e) \leq|e|^{2} / 2$ for all $e \in \mathbf{R}^{n}$,

$$
\begin{aligned}
0 & \geq \lim _{q \rightarrow \infty} \frac{\tilde{K}\left(z_{0 b_{0}} ; z_{b_{q}}^{q}\right)}{d_{q}^{2}}+\liminf _{q \rightarrow \infty} \frac{\delta}{d_{q}^{2}} \mathcal{Q}\left(z^{q}-z_{0}\right) \\
& =\lim _{q \rightarrow \infty} \frac{\tilde{K}\left(z_{0 b_{0}} ; z_{b_{q}}^{q}\right)}{d_{q}^{2}}+\liminf _{q \rightarrow \infty} \frac{\delta}{d_{q}^{2}}\left[D\left(z^{q}-z_{0}\right)-V\left(x_{q}\left(t_{0}\right)-x_{0}\left(t_{0}\right)\right)\right] \\
& \geq \lim _{q \rightarrow \infty} \frac{\tilde{K}\left(z_{0 b_{0}} ; z_{b_{q}}^{q}\right)}{d_{q}^{2}}+\frac{\delta}{2}-\frac{\delta}{2} \limsup _{q \rightarrow \infty} \frac{\left|x_{q}\left(t_{0}\right)-x_{0}\left(t_{0}\right)\right|^{2}}{d_{q}^{2}} \\
& =\lim _{q \rightarrow \infty} \frac{\tilde{K}\left(z_{0 b_{0}} ; z_{b_{q}}^{q}\right)}{d_{q}^{2}}+\frac{\delta}{2}-\frac{\delta}{2} \limsup _{q \rightarrow \infty} \frac{\left|\Psi_{0}\left(b_{q}\right)-\Psi_{0}\left(b_{0}\right)\right|^{2}}{d_{q}^{2}} \\
& =\lim _{q \rightarrow \infty} \frac{\tilde{K}\left(z_{0 b_{0}} ; z_{b_{q}}^{q}\right)}{d_{q}^{2}}+\frac{\delta}{2}-\frac{\delta}{2} \limsup _{q \rightarrow \infty}\left|\int_{0}^{1} \Psi_{0}^{\prime}\left(b_{0}+\lambda\left[b_{q}-b_{0}\right]\right)\left(\frac{b_{q}-b_{0}}{d_{q}}\right) d \lambda\right|^{2} \\
& =\lim _{q \rightarrow \infty} \frac{\tilde{K}\left(z_{0 b_{0}} ; z_{b_{q}}^{q}\right)}{d_{q}^{2}}+\frac{\delta}{2}-\frac{\delta}{2}\left|\Psi_{0}^{\prime}\left(b_{0}\right) \beta_{0}\right|^{2} \\
& =\lim _{q \rightarrow \infty} \frac{\tilde{K}\left(z_{0 b_{0}} ; z_{b_{q}}^{q}\right)}{d_{q}^{2}}+\frac{\delta}{2}-\frac{\delta}{2}\left|y_{0}\left(t_{0}\right)\right|^{2} .
\end{aligned}
$$

With this in mind and (14), if we suppose that $w_{0 \beta_{0}} \equiv(0,0,0)$, then $\delta$ would not be positive, which is not the case, and this establishes (13). Now, let us prove that

$$
\dot{y}_{0}(t)=f_{x}\left(t, x_{0}(t), u_{0}(t)\right) y_{0}(t)+f_{u}\left(t, x_{0}(t), u_{0}(t)\right) v_{0}(t) \quad(\text { a.e. in } T) .
$$

Note that, for all $q \in \mathbf{N}$

$$
\dot{y}_{q}(t)=A_{q}(t) y_{q}(t)+B_{q}(t) v_{q}(t) \quad(\text { a.e. in } T)
$$

where

$$
A_{q}(t)=\int_{0}^{1} f_{x}\left(t, x_{0}(t)+\lambda\left[x_{q}(t)-x_{0}(t)\right], u_{0}(t)+\lambda\left[u_{q}(t)-u_{0}(t)\right]\right) d \lambda
$$




$$
B_{q}(t)=\int_{0}^{1} f_{u}\left(t, x_{0}(t)+\lambda\left[x_{q}(t)-x_{0}(t)\right], u_{0}(t)+\lambda\left[u_{q}(t)-u_{0}(t)\right]\right) d \lambda .
$$

As

$$
A_{q}(\cdot) \stackrel{L^{\infty}}{\longrightarrow} f_{x}\left(\cdot, x_{0}(\cdot), u_{0}(\cdot)\right), \quad B_{q}(\cdot) \stackrel{L^{\infty}}{\longrightarrow} f_{u}\left(\cdot, x_{0}(\cdot), u_{0}(\cdot)\right), \quad y_{q} \stackrel{\mathrm{u}}{\longrightarrow} y_{0}, \quad v_{q} \stackrel{L^{1}}{\longrightarrow} v_{0},
$$

all on $T$, it follows that

$$
\dot{y}_{q}(\cdot) \stackrel{L^{1}}{\rightarrow} f_{x}\left(\cdot, x_{0}(\cdot), u_{0}(\cdot)\right) y_{0}(\cdot)+f_{u}\left(\cdot, x_{0}(\cdot), u_{0}(\cdot)\right) v_{0}(\cdot) \text { on } T .
$$

By Lemma $3, \dot{y}_{q} \stackrel{L^{1}}{\longrightarrow} \sigma_{0}=\dot{y}_{0}$ on $T$. Then, (19) is verified.

Now, we claim that

i. $\varphi_{\alpha x}\left(t, x_{0}(t), u_{0}(t)\right) y_{0}(t)+\varphi_{\alpha u}\left(t, x_{0}(t), u_{0}(t)\right) v_{0}(t) \leq 0$ (a.e. in $T, \alpha \in \mathcal{I}_{a}\left(t, x_{0}(t), u_{0}(t)\right)$ ).

ii. $\varphi_{\beta x}\left(t, x_{0}(t), u_{0}(t)\right) y_{0}(t)+\varphi_{\beta u}\left(t, x_{0}(t), u_{0}(t)\right) v_{0}(t)=0$ (a.e. in $T, \beta \in S$ ).

As one can easily verify, (i) and (ii) above are obtained if one simply copies the proofs from (27) to (29) of [25].

Consequently, from (11), (19), (i) and (ii), above, it follows that $w_{0 \beta_{0}} \in \mathcal{Y}\left(z_{0 b_{0}}\right)$. This fact together with (13) contradict hypothesis (iv) of Theorem 1.

Case (2): Now, assume that the sequence $\left\{\left(b_{q}-b_{0}\right) / d_{q}\right\}$ is unbounded. Then,

$$
\lim _{q \rightarrow \infty}\left|\frac{b_{q}-b_{0}}{d_{q}}\right|=+\infty
$$

In this case, if one copies the proofs from (31) to (38) of [25], then one obtains that for some $\bar{\beta}_{0} \in \mathbf{R}^{p}$ with $\left|\bar{\beta}_{0}\right|=1$,

a. $\Psi_{i}^{\prime}\left(b_{0}\right) \bar{\beta}_{0}=0(i=0,1)$.

b. $J^{\prime \prime}\left(z_{0 b_{0}} ; 0_{\bar{\beta}_{0}}\right) \leq 0$.

Consequently, (a) and (b) above contradict hypothesis (iv) of Theorem 1.

\section{Conclusions}

In this paper, we have obtained two sufficiency theorems for a parametric and a nonparametric problems of Bolza having nonlinear dynamics, variable initial and final states and nonlinear inequality and equality mixed time-state-control constraints. The proposed optimal controls need not be continuous but also purely measurable. In the nonparametric problem, the initial and final states are not only variable but also completely free, all the crucial sufficiency hypotheses are included in the theorems and, in the parametric sufficiency theorem, the deviation around the optimal cost, can be measured by a proportion of a function involving several functionals playing similar roles of the square of some norms. The algorithm of sufficiency used to prove one of the main results of the article is self-contained in the sense that it is independent of classical techniques used to obtain sufficiency of problems having a similar degree of generality as the one studied in this work. On the other hand, some future directions of research can be visualized by applying this method of sufficiency. Concretely, we conjecture that Corollary 1 can be proved directly, that is, without invoking the theorem of the parametric problem. The only issue that must be addressed is that the sets $\mathcal{B}_{i}(i=0,1)$ appearing in the corollary should be manifolds determined by some $C^{2}$ functions $\Phi_{-i}(i=-1,0)$, satisfying the relations $x\left(t_{-i}\right)=\Phi_{-i}\left(x\left(t_{i+1}\right)\right)(i=-1,0)$. We also conjecture that a parallel version of Corollary 1 can be derived directly with the sets $\mathcal{B}_{i}(i=0,1)$ being any sets and they need not have to be subsets of any manifold. Once again, another issue that possibly arises is that we have to diminish the class of admissible processes by requiring that the strategies be of class $C^{2}$ instead of being absolutely continuous on the underlying interval of time.

Funding: This research was funded by Dirección General de Asuntos del Personal Académico, DGAPA-UNAM, by the project PAPIIT-IN102220. 


\section{Data Availability Statement: Not applicable.}

Acknowledgments: The author thanks the Dirección General de Asuntos del Personal Académico, Universidad Nacional Autónoma de México, for the financial support provided by the project PAPIIT-IN102220. Additionally, the author thanks the three anonymous referees for the encouraging comments made in their reviews.

Conflicts of Interest: The author declares no conflict of interest.

\section{References}

1. Malanowski, K. Sufficient optimality conditions for optimal control subject to state constraints. SIAM J. Control Optim. 1997, 35, 205-227. [CrossRef]

2. Malanowski, K.; Maurer, H. Sensitivity analysis for parametric control problems with control-state constraints. Comput. Optim. Appl. 1996, 5, 253-283. [CrossRef]

3. Malanowski, K.; Maurer, H.; Pickenhain S. Second order sufficient conditions for state-constrained optimal control problems. J. Optim. Theory Appl. 2004, 123, 595-617. [CrossRef]

4. Maurer, H. First, and second order sufficient optimality conditions in mathematical programming and optimal control. In Mathematical Programming at Oberwolfach; Springer: Berlin/Heidelberg, Germany, 1981; Volume 14, pp. 163-177.

5. Maurer, H. Sufficient conditions and sensitivity analysis for economic control problems. Ann. Oper. Res. 1999, 88, 3-14. [CrossRef]

6. Maurer, H.; Oberle, H.J. Second order sufficient conditions for optimal control problems with free final time: The Riccati approach. SIAM J. Control Optim. 2002, 41, 380-403. [CrossRef]

7. Maurer, H.; Pickenhain, S. Second order sufficient conditions for control problems with mixed control-state constraints. J. Optim. Theory Appl. 1995, 86, 649-667. [CrossRef]

8. Loewen, P.D. Second-order sufficiency criteria and local convexity for equivalent problems in the calculus of variations. J. Math Anal. Appl. 1990, 146, 512-522. [CrossRef]

9. Rosenblueth, J.F. Variational conditions and conjugate points for the fixed-endpoint control problem. IMA J. Math. Control Inf. 1999, 16, 147-163. [CrossRef]

10. Osmolovskii, N.P. Second order sufficient conditions for an extremum in optimal control. Control Cybern. 2002, 31, 803-831.

11. Osmolovskii, N.P. Second-order sufficient optimality conditions for control problems with linearly independent gradients of control constraints. ESAIM Control. Optim. Calc. Var. 2012, 18, 452-482. [CrossRef]

12. Stefani, G.; Zezza, P.L. Optimality conditions for a constrained optimal control problem. SIAM J. Control Optim. 1996, 34, 635-659. [CrossRef]

13. Hestenes, M.R. Calculus of Variations and Optimal Control Theory; John Wiley: New York, NJ, USA, 1966.

14. Milyutin, A.A.; Osmolovskii, N.P. Calculus of Variations and Optimal Control; American Mathematical Society: Providence, RI, USA, 1998.

15. Stefani, G.; Zezza, P.L. Constrained regular LQ-control problems. SIAM J. Control Optim. 1997, 35, 876-900. [CrossRef]

16. Maurer, H.; Pesh, H.J. Solution differentiability for parametric nonlinear control problems with control-state constraints. J. Optim. Theory Appl. 1995, 86, 285-309. [CrossRef]

17. Malanowski, K. Two norm approach in stability and sensitivity analysis of optimization and optimal control problems. Adv. Math. Sci. Appl. 1993, 2, 397-443.

18. Cortez, K.L.; Rosenblueth, J.F. Normality and uniqueness of Lagrange multipliers. Discret. Contin. Dyn. Syst. 2018, 38, 3169-3188. [CrossRef]

19. Cortez, K.L.; Rosenblueth, J.F. The broken link between normality and regularity in the calculus of variations. Syst. Control Lett. 2019, 124, 27-32. [CrossRef]

20. Rosário de Pinho, M.D.; Rosenblueth, J.F. Mixed constraints in optimal control: An implicit function theorem approach. IMA J. Math. Control Inf. 2007, 24, 197-218. [CrossRef]

21. Alt, W.; Felgenhauer, U.; Seydenschwanz, M. Euler discretization for a class of nonlinear optimal control problems with control appearing linearly. Comput. Optim. Appl. 2018, 69, 825-856. [CrossRef]

22. Osmolovskii, N.P.; Veliov, V.M. Metric sub-regularity in optimal control of affine problems with free end state. ESAIM Control Optim. Calc. Var. 2019. [CrossRef]

23. Sánchez Licea, G. Sufficiency for purely essentially bounded optimal controls. Symmetry 2020, 12, 238. [CrossRef]

24. Lennon, O.N.; Áine, B. Piecewise-constant optimal control strategies for controlling the outbreak of COVID-19 in the Irish population. Math. Biosci. 2020, 330, 108496.

25. Sánchez Licea, G. Sufficiency for singular trajectories in the calculus of variations. AIMS Math. 2019, 5, 111-139. [CrossRef] 